responses to auditory and tactile stimuli are reported from Bambino Gesu Children's Hospital, Rome, Italy. An excessive startle response caused symmetric jumping of limbs, arms more than legs. Spontaneous attacks developed in 4, particularly in sleep. A family history of epilepsy or febrile convulsions was elicited in 5. Remissions occurred in 4-14 months, spontaneously or in response to valproate. EEGs showed short generalized spike- or polyspike-and-wave discharges. (Ricci S et al. Reflex myoclonic epilepsy in infancy: A new age-dependent idiopathic epileptic syndrome related to startle reaction. Epilepsia April 1995;36:342-348). (Reprints: Dr S Ricci, Section of Neurophysiology, Bambino Gesu Children's Hospital, IRCCS, Piazza S Onofrio 4, 00165 Rome, Italy).

COMMENT. In addition to the reflex nature of these attacks, the abnormal EEG appears to distinguish this syndrome from benign myoclonic epilepsy in infancy. Therapy with anticonvulsants seemed justified when attacks occurred spontaneously and frequently, from 5 to 20 times daily.

\title{
READING EPILEPSY
}

The electroclinical manifestations and natural history of reading epilepsy (RE) in 20 patients diagnosed between 1949 and 1989 are reported from the Mayo Clinic, Rochester, Minnesota. Age at onset ranged from 10 to 46 years (median 17 years). Juvenile myoclonic epilepsy occurred in 4 , and a positive family history for epilepsy in 4, with RE in 1 . Seizures were myoclonic, involving orofacial and jaw muscles, and the upper limbs also in 5 . Generalized tonic-clonic seizures occurred at least once in 16. The EEG showed generalized spike or spike-and wave discharges in 15 cases and left hemisphere discharges in 5. RE was persistent into late adult life but not progressive; it responded to valproic acid. Higher cognitive processes acting as trigger mechanisms other than reading included calculation in 6 , speaking under stress in 5, writing in 2, and playing chess in 1. (Radhakrishnan K, Silbert PL, Klass DW. Reading epilepsy. An appraisal of 20 patients diagnosed at the Mayo Clinic, Rochester, Minnesota, between 1949 and 1989, and delineation of the epileptic syndrome. Brain Feb 1995;118:75-89). (Respond: Donald W Klass MD, Section of Electroencephalography, Mayo Clinic, 200 First Street SW, Rochester, MN 55905).

COMMENT. The authors dedicate their article to Dr Reginald G Bickford on his 81st birthday and we add our congratulations! Bickford (1954) and Bickford, Klass et al (1956) first described the syndrome of reading epilepsy and stressed the importance of precipitating factors in the mechanism of seizures and EEG epileptiform discharges in general. Christie S (1988) found a combination of factors involved in the precipitation of reading epilepsy: saccadic eye movements, articulation, and difficulty of linguistic content. Bickford had alluded to the degree of difficulty of reading matter in his original article. (See Progress in Pediatric Neurology I, 1991, p45).

\section{PREVENTION OF EPILEPSY AND COMPLICATIONS}

Prevention of epilepsy and its consequencies is discussed in a special article from the Department of Neurology, School of Medicine, University of Virginia, Charlottesville. Prevention should apply at various levels: 1 . epileptogenesis may be prevented by a) avoidance of premature birth, in 
utero infection, and anoxia; b) prevention of febrile convulsions and their complications; c) avoidance of head trauma by wearing helmets when bicycling and banning of boxing as high school sport; 2. ictogenesis, individual epileptic seizures, may be prevented by identifying and controlling precipitating or provocative factors such as drugs and alcohol, sleep deprivation, AED withdrawal or poor compliance, and photic stimulation; 3 ) neurologic consequencies can be prevented by early and optimal treatment of seizures and avoidance of status epilepticus; 4) psychosocial consequencies could be lessened by public education, removal of stigmata, and improvement in the quality of life; and 5) treatment consequencies involving anticonvulsant side-effects, teratogenicity, and cognitive and other deficits resulting from overzealous surgical resections. (Dreifuss FE. Prevention as it pertains to epilepsy. Arch Neurol April 1995;52:363-366). (Reprints: Dr Dreifuss, Department of Neurology, School of Medicine, University of Virginia, Charlottesville, VA 22908).

COMMENT. Prevention in the management of epilepsy has been sadly neglected, and Dr Dreifuss in his synopsis draws attention to many adverse factors and aspects of etiology and treatment that could be avoided or corrected when addressed appropriately. Additional problems, especially important to the adolescent and young adult, include the permission to drive an automobile and the risk of accidents. Young drivers account for one half those with seizures at the wheel, and a complex partial seizure, usually without aura, is the most common pattern associated with accidents. Those with auras are significantly less likely to lead to accidents. Males, 19 to 30 years, in higher socioeconomic classes, form the majority continuing to drive without adequate seizure control. The monitoring of young male drivers with complex partial seizures should be close and frequent, including serum drug levels to check compliance and adequate AED dosage. (Ped Neur Briefs Oct 1987; see Progress in Pediatric Neurology I, 1991, p129-131).

The stress and anxieties associated with the first year away at college are additional reasons for relapse and recurrence of seizures in the young adult. Seizures may be prevented by counselling and by modification of medications to cover this period.

The need for further training of physicians in preventive measures is stressed in the following article from Wales regarding the management of status epilepticus in hospitalized patients.

\section{MANAGEMENT OF STATUS EPILEPTICUS}

The care given to 8 children with epilepsy admitted with convulsive status epilepticus during 1990 is evaluated at the University Hospital of Wales, Cardiff. All had significant learning disabilities. In 17 admissions, eye-witness accounts of the seizure leading to admission were not recorded in 5 , and were poorly described in 11 . Of 9 convulsive episodes persisting on admission, 6 had been treated with rectal diazepam in the home. All children were on maintenance therapy for previously diagnosed epilepsy, but only one was recorded as compliant. AED dosage was adjusted after 8 of the episodes, less than half the total. Prevention of further episodes after discharge was not considered in 8 cases. The availability of rectal diazepam in the home was ignored for most admissions, and parents had not been instructed in its optimal usage. Average length of stay was 2 days. (Matthes JWA, Wallace SJ. Convulsive status epilepticus in children treated for epilepsy: an assessment of 